\title{
First Draft Genome of Two Candida haemulonii Strains from Pediatric Patients with Candidemia in Brazil
}

Luiza Souza Rodrigues ${ }^{1,2}$, Rajesh Kumar Gazara ${ }^{3}$, Hemanoel Passarelli ${ }^{3}$, Robson Francisco de Souza ${ }^{4}$, Thiago Motta Venancio ${ }^{3 \#}$ and Libera Maria Dalla Costa ${ }^{1 \#}$

${ }_{1}^{1}$ Faculdades Pequeno Príncipe e Instituto de Pesquisas Pelé Pequeno Príncipe (FPP/IPPPP), Curitiba-PR, Brazil; ${ }^{2}$ Centro Universitário Autônomo do Brasil (UniBrasil), Curitiba-PR, Brazil; ${ }^{3}$ Lab. de Química e Função de Proteínas e Peptídeos, CBB/UENF, Campos dos Goytacazes-RJ, Brazil; ${ }^{4}$ Laboratório de Estrutura e Evolução de Proteínas (LEEP)/ICB/USP, São Paulo-SP, Brazil.

\# Co-Corresponding authors

\section{INTRODUCTION}

Members of the Candida haemulonii species complex (C.haemulonii, C. duobushaemulonii and C. haemulonii var. vulnera) are emerging yeasts associated to bloodstream and other invasive infections. Considered as oportunistic pathogens they are phylogenetically related to $C$. auris, which shares a propensity for multidrug resistance. However, genomic information of $C$. haemulonii remains scarse. We describe the draft genome sequencing of two $C$. haemulonii strains isolated from pediatric patients with candidemia at a tertiary pediatric hospital of South of Brazil.

\section{METHOD}

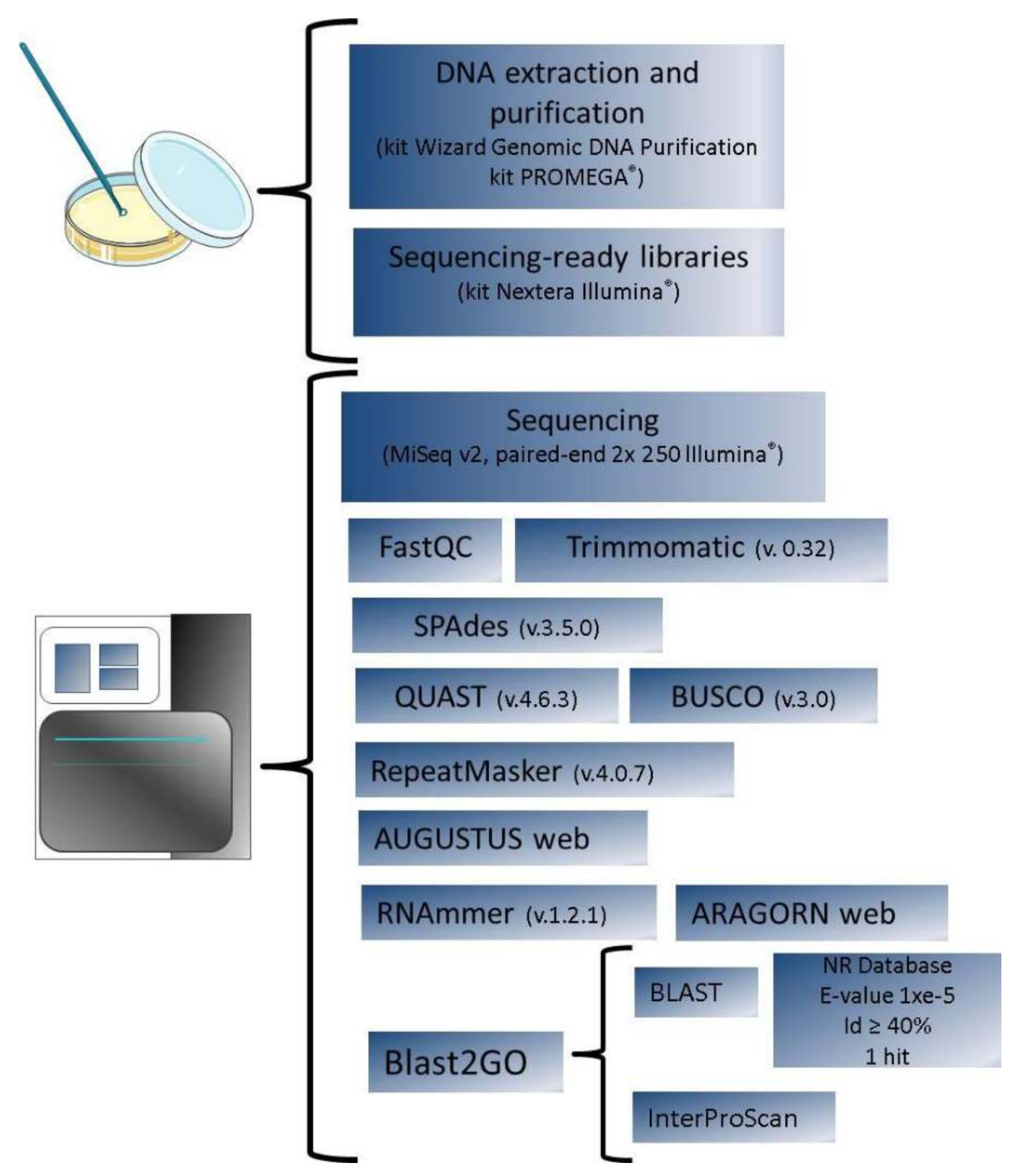

Muro MD, Motta Fde A, Burger M, Melo AS, Dalla-Costa LM. Echinocandin resistance in two Candida haemulonii isolates from pediatric patients. J Clin Microbiol. 2012;50(11):3783-5.

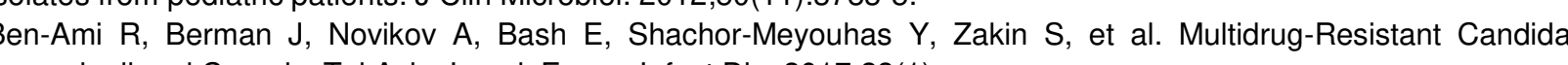

man A, et al. Candida auris: a Review of the Literature. Clin Microbiol Rev. 2018:31(1).

\section{RESULTS}

a. Top BLAST Hits Candida Species Distributions

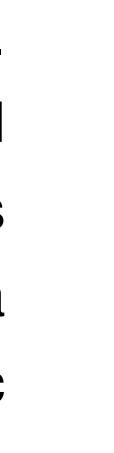 \\ i
}

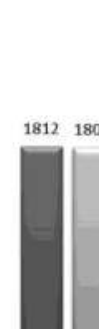

b.
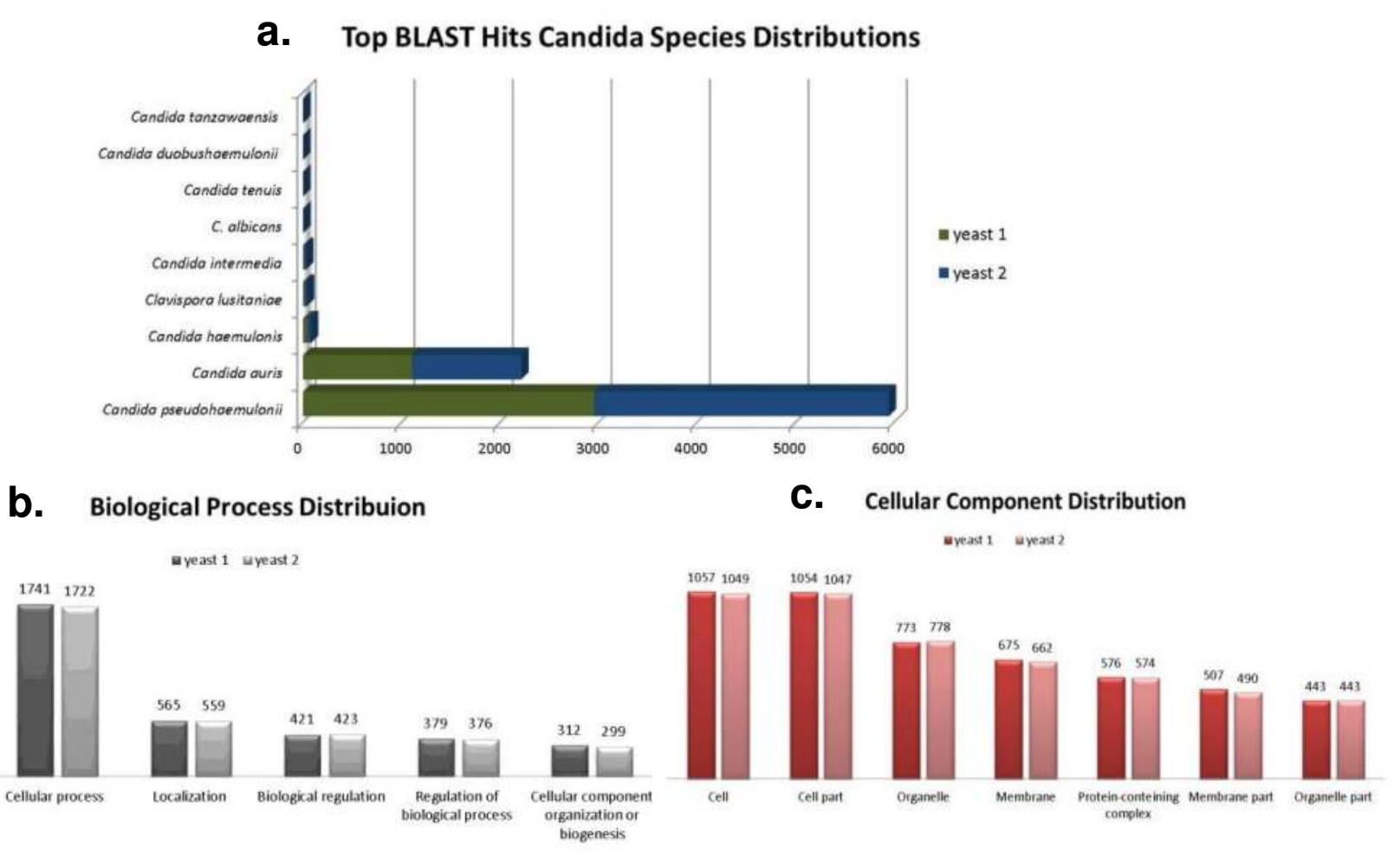

d. Molecular Functions Distribution

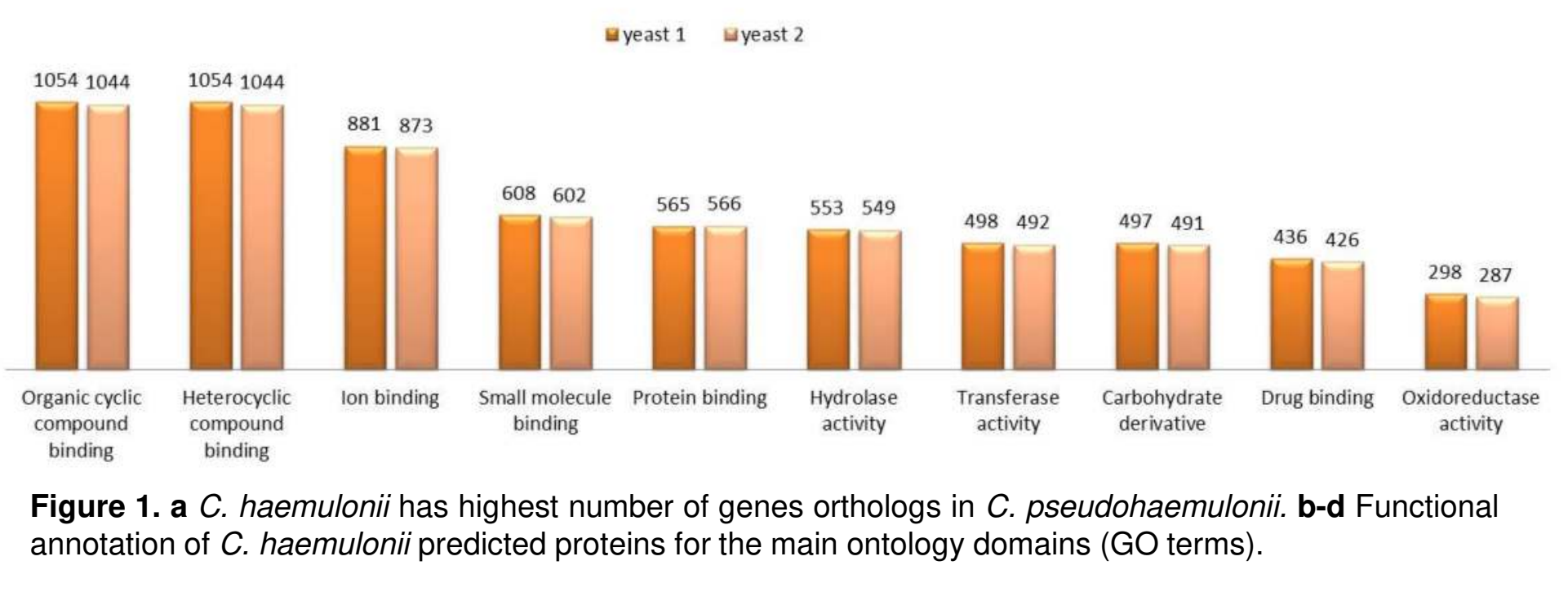

The draft genomes comprised 352 and 388 scaffolds, with a N50 of $78,004 \mathrm{bp}$ and $75,117 \mathrm{bp}$, and largest scaffolds with 393,866 bp and 251,044 bp. Total genome sizes were $13,21 \mathrm{Mb}$ and $13,26 \mathrm{Mb}$, with an average $\mathrm{G}+\mathrm{C}$ content of $44 \%$. BUSCO analysis (Saccharomycetales dataset as reference, 1,771 genes) indicated that the assemblies are $94.6 \%$ and $94.5 \%$ complete. Less than $2.0 \%$ of the sequences were masked. The complete genomes sequences contain genes for 6 rRNAs, 175 and 173 tRNAs. AUGUSTUS resulted in 5,484 and 5,508 proteins-coding genes and functional annotations were done in Blast2GO resulted in 3,690 and 3,682 sequences with GO terms.

\section{CONCLUSION}

The data provided here will constitute a powerful tool for fungal biology and virulence studies, also to better understand of the phylogenetic relationships to other Candida species, such as the emerging pathogen $C$. auris. 